\title{
Resection of a tubular adenoma in the distal common bile duct through percutaneous choledochoscopy
}

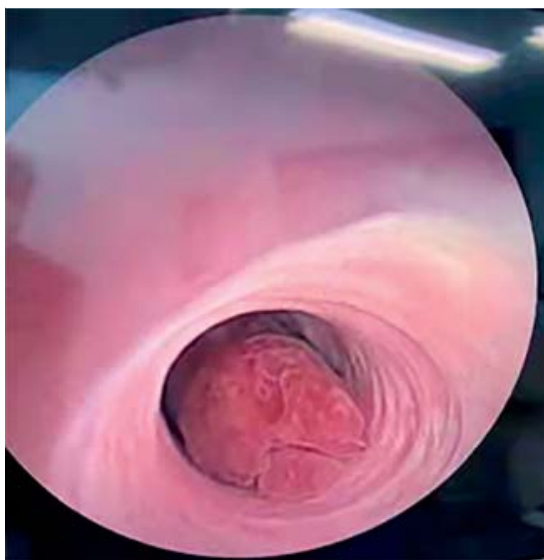

- Fig. 1 Accidentally detected neoplasm in the common bile duct of a 59-year-old man.

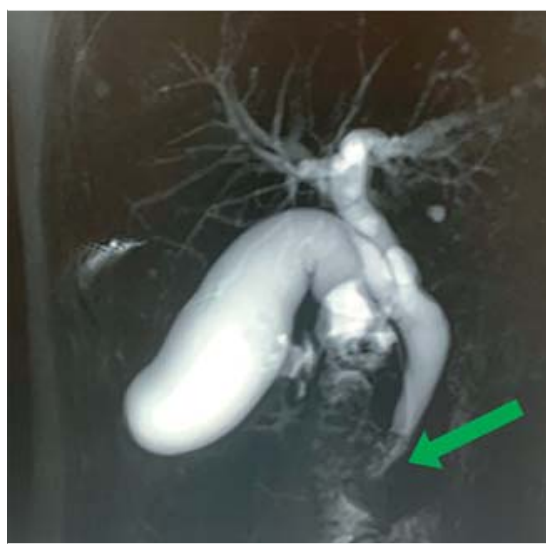

- Fig. 2 Magnetic resonance cholangiopancreatography showed a filling defect in the common bile duct without invasion of surrounding organs.

A 59-year-old man was admitted to our hospital with a neoplasm in the distal common bile duct ( $\mathbf{F i g . 1}$ ), which had been detected during laparoscopic exploration for biliary stone in a local hospital 2 months earlier. Magnetic resonance cholangiopancreatography showed a filling defect without invasion of surrounding organs ( $>$ Fig.2). Combining the result of multidisciplinary discussion and the wishes of the patient, percutaneous

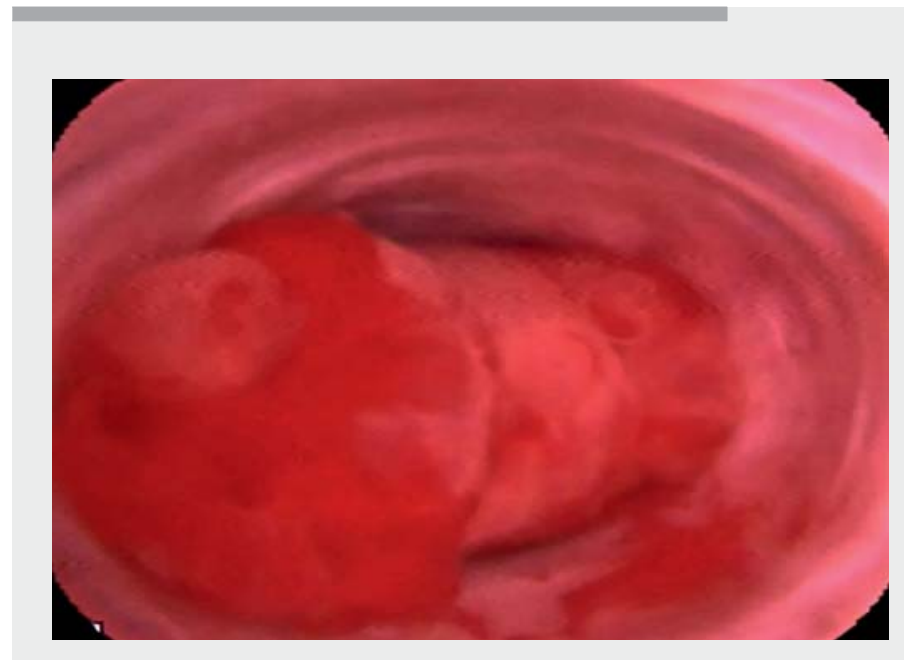

$\checkmark$ Video 1 The pedicled lesion was reached using percutaneous choledochoscopy through the sinus tract formed by the T-tube. A hot snare was used to resect the lesion under direct visualization. At the end, a clean and flat wound bed was left after the resection.

choledochoscopic treatment through the T-tube tract was performed for him ( Video 1).

After identification of the pedunculated neoplasm ( $\mathbf{F i g . 3 a ) , ~ a ~ h o t ~ s n a r e ~ w a s ~}$ used to resect the lesion under direct

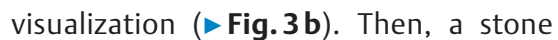
basket was used to extract the lesion ( Fig.3c). The wound was clean and flat, without residual tumor ( $>$ Fig. 3 d). The whole process took about $10 \mathrm{~min}$ utes and there was no complication. Pathologic analysis showed the neoplasm to be a tubular adenoma ( $>$ Fig.4). With unknown malignant potential and the risk of causing obstructive jaundice, benign adenomas of the common bile duct are suggested to be treated by surgery at present time. However, the surgical procedure is extensive and complex. In recent years, peroral cholangioscopic procedures have been reported for treating benign neoplasms in the biliary tract, ensuring the minimum of injury and a good outcome [1]. As far as we know, the present case is the first report re- garding percutaneous choledochoscopy for resection of a benign biliary neoplasm. Our experience indicates that percutaneous choledochoscopy could be an alternative for treatment of benign biliary tumors. Given the risk of recurrence of adenomas, regular follow-up is critical. The safety and effectiveness of this endoscopic method requires confirmation by further studies.

Endoscopy_UCTN_Code_TTT_1AR_2AJ

\section{Competing interests}

The authors declare that they have no conflict of interest.

\section{Funding}

1.3.5 project for disciplines of excellence Clinical Research Incubation Project, West China Hospital, Sichuan University $20 \mathrm{HXFH} 016$ 

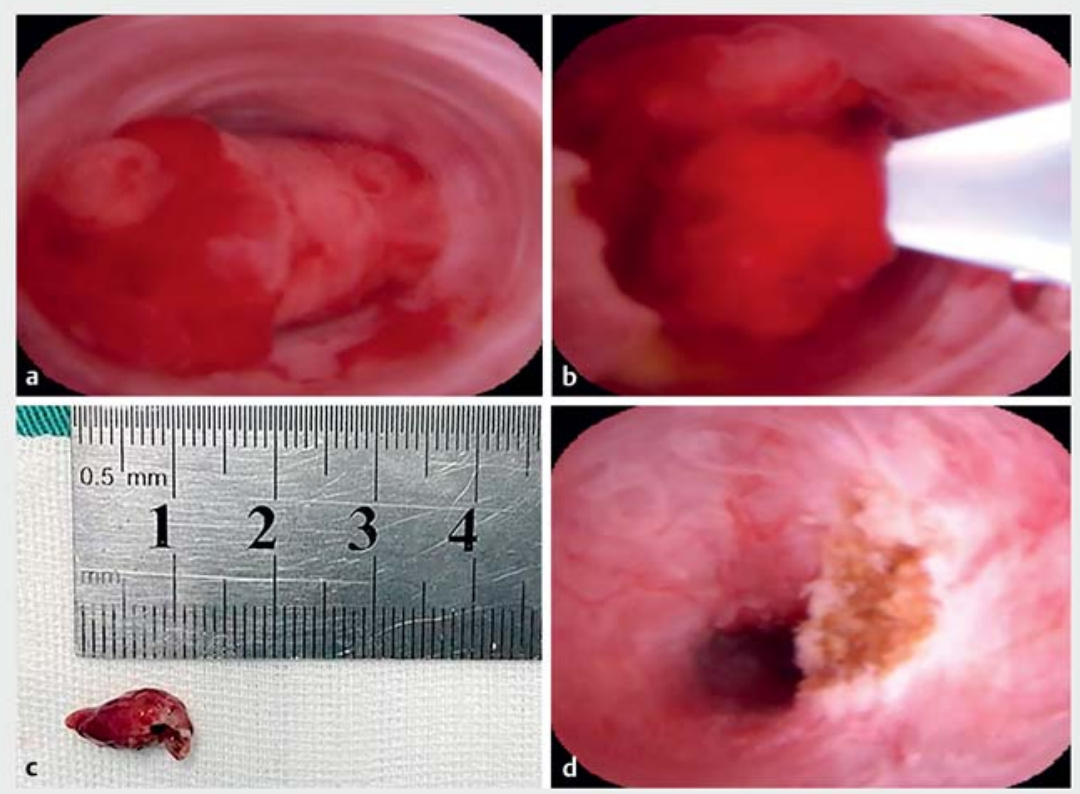

- Fig. $\mathbf{3}$ a-d The process of resection. a The pedunculated neoplasm. b A hot snare was used to resect the lesion under direct visualization. $\mathbf{c}$ The resected specimen. $\mathbf{d}$ A clean and flat wound bed left after the resection.

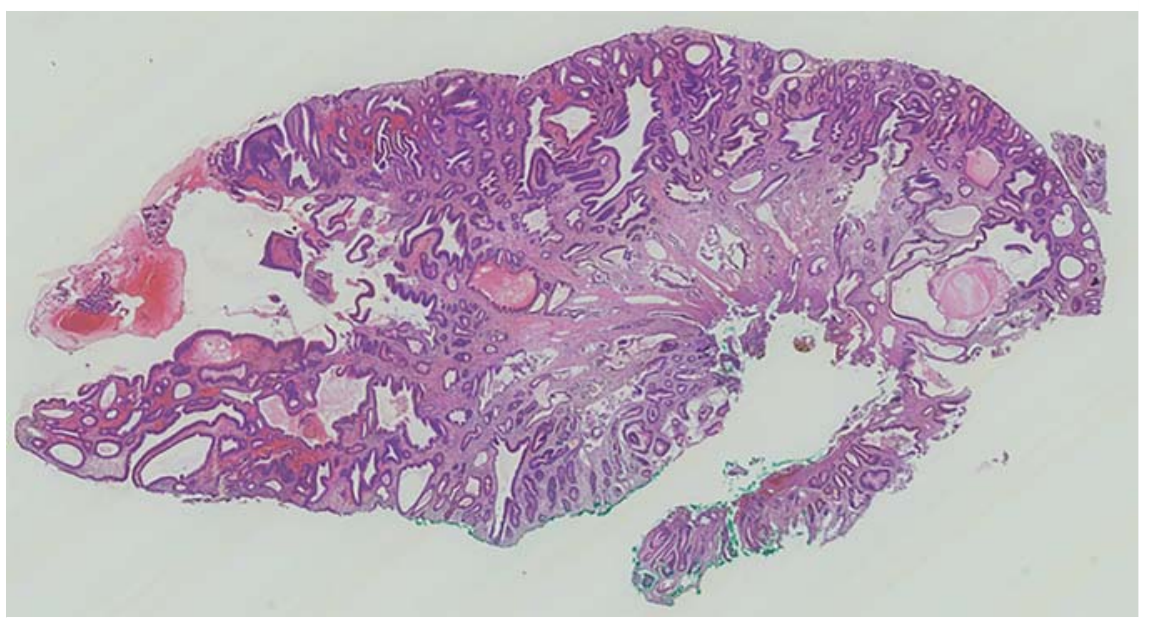

Fig. 4 H\&E staining indicated the lesion was tubular adenoma.
The authors

Long He*, Shuai Bai", Liansong Ye, Bing Hu

Department of Gastroenterology, West China

Hospital, Sichuan University, Chengdu, Sichuan, China

\section{Corresponding author}

\section{Bing Hu, MD}

Department of Gastroenterology, West China Hospital, No. 37, Guo Xue Alley, Wuhou District, Chengdu City, Sichuan Province, P. R. China hubingnj@163.com

\section{Reference}

[1] Bendezu-Garcia RA, Andujar-Murcia X, Loras C. Endoscopic removal of tubulovillous adenoma with high grade focal dysplasia in the distal common bile duct. Endoscopy 2019; 51: E319-E320

\section{Bibliography}

Endoscopy 2022; 54: E405-E406

DOI $10.1055 / \mathrm{a}-1541-7246$

ISSN 0013-726X

published online 8.9.2021

(c) 2021. Thieme. All rights reserved.

Georg Thieme Verlag KG, Rüdigerstraße 14,

70469 Stuttgart, Germany

\section{ENDOSCOPY E-VIDEOS}

https://eref.thieme.de/e-videos

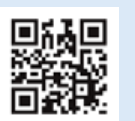

Endoscopy E-Videos is an open access online section, reporting on interesting cases and new techniques in gastroenterological endoscopy. All papers include a high quality video and all contributions are freely accessible online. Processing charges apply (currently EUR 375), discounts and wavers acc. to HINARI are available.

This section has its own submission website at

https://mc.manuscriptcentral.com/e-videos

\footnotetext{
${ }^{*}$ Co-first authors
} 